\title{
Zur Kenntnis der Carbinolverbindungen des Triphenylmethans und seiner Derivate
} von

\section{J. Herzig und P. Wengraf.}

Aus dem I. chemischen Laboratorium der k. k. Universität in Wien.

(Vorgelegt in der Sitzung am 23. Mai 1901.)

Vor einiger Zeit haben Herzig und Smoluchowski das Aurin studiert und dabei zwei isomere Acetylverbindungen erhalten, die sich von der Carbinolformel des Aurins ableiten lassen. Da sich die Hydroxylgruppe in keinem der beiden Fälle nachweisen ließ, so gelangten die genannten Autoren nach einer ausführlichen Discussion aller Möglichkeiten $z u$ einer hypothetischen Constitutionsformel dieser Substanzen, welche den Acetylaurinen keinen carbinolartigen Charakter zuwies und die Isomerie in einer der Baeyer'schen Hydrophtalsäureisomerie analogen Form erklären lässt.

Wir hätten demnach für die beiden Körper folgende Formeln:

I.

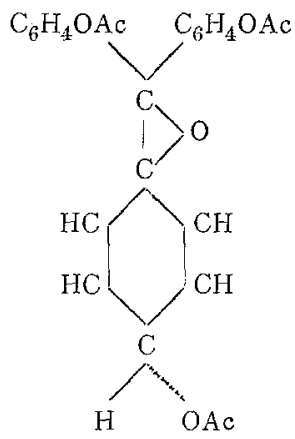

II.

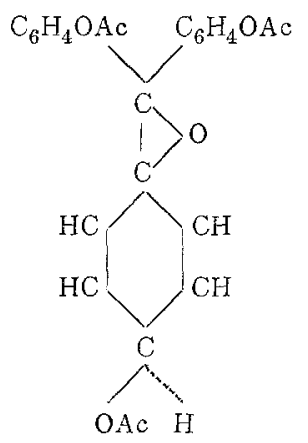

1 Monatshefte für Chemie, Bd. XV, S. 73; Bd. XVII, S. 191. 
wobei wir uns nach der Herzig-Smoluchowski'schen Schreibweise richten, die durch eine gestrichelte und eine ausgezogene Linie andeutet, dass die Acetoxyle das einemal hinter, das anderemal vor der Papierebene liegen sollen.

Über die Function des Carbinolhydroxyls war zu jener Zeit noch wenig bekannt. Wohl hatte Döbner schon das Acetylbenzaurin, Hemilian das Triphenylcarbinol selbst studiert, allein beide hatten sich darauf beschränkt, zu constatieren, dass das Carbinolhydroxyl der Esterificierung nur schwer oder gar nicht zugänglich sei.

Es wurden deshalb bereits in der genannten Aurinarbeit Versuche in Aussicht gestellt, die sich über angrenzende Gebiete verbreiten sollten, um die Natur und die Functionen der Hydroxylgruppe in derartig complicierten Carbinolverbindungen zu ermitteln.

Zunächst hat sich nun die Rosolsäure bei unseren Versuchen als ein vollkommenes Analogon des Aurins erwiesen, indem auch hier die Bildung zweier isomerer Verbindungen wahrscheinlich wurde, von denen die eine gut studiert werden konnte.

Auch bei dem von Döbner dargestellten Benzaurin konnten Anzeichen für eine ähnliche Isomerie beobachtet werden. Außerdem wurde die Analogie hier noch weiter verfolgt, insoferne, als die leichte Reductionsfähigkeit des erhaltenen Acetylbenzaurins in essigsaurer Lösung zum Acetylleukobenzaurin constatiert werden konnte.

Da aber in beiden Fällen die Hydroxylgruppe sich vollkommen indifferent verhielt, so war von diesen Verbindungen aus eine Aufklärung nicht zu erwarten und wir haben uns daher dem Studium der Substanzen zugewendet, welche als Beweise für die Hydroxylgruppe in den Carbinolverbindungen gelten durften.

Es waren dies hauptsächlich die Alkyläther, die O.F i s cher ${ }^{1}$ beim Carbinol des Bittermandelölgrüns und Rosenstieh12 bei dem des Krystallvioletts dargestellt haben. Diese Äther erheischten eine neuerliche Bearbeitung, weil ihre Natur als

1 Lieb. Ann., 206, 132.

2 Comptes rendus, 120 , S. 192, 264, 331. 
Alkyläther nicht durch eine Zeisel'sche Bestimmung controliert war.

Die Darstellung des Fischer'schen Äthers gelang uns fürs erste gar nicht, weil immer Verfärbung und Zersetzung eintraten und wir im Vacuum zu keiner Constanz des Gewichtes gelangen konnten. Erst durch eine Beobachtung O. Fischers, die er uns gütigst mittheilte, gelang es uns, den Methyläther des Bittermandelölgrüns darzustellen und dessen Zusammensetzung durch eine Methoxylbestimmung festzulegen.

Die oben erwähnte Beobachtung $O$. Fischers, welche seither übrigens auch publiciert wurde, ${ }^{1}$ beruht auf einem sehr merkwürdigen Verhalten dieses Äthers. Derselbe ist närnlich gegen Säure außerordentlich empfindlich, und die Darstellung und das Trocknen gelingt nur dann, wenn man mit großer Sorgfalt jede Spur von Säuren ausschließt.

Mit diesen Erfahrungen ausgerüstet konnten wir auch den Ather des Krystallviolettes nach Rosenstiehl darstellen, und auch hier ergab die Äthoxylbestimmung ein Resultat, welches über die Natur dieser Verbinđung als Alkyläther keinen Zweifel bestehen lässt.

Durch diese Thatsache verliert die Möglichkeit, dass die Hydroxylgruppe in den oben erwähnten Acetylverbindungen zwar vorhanden, aber durch ihre Lage in ihrer Reactionsfähigkeit behindert sei, sehr an Wahrscheinlichkeit, und man sieht sich umsomehr genöthigt, an eine der Carbinolformel tautomere Form zu denken. Jedenfails ist ohneweiters nicht einzusehen, wodurch die Hydroxylgruppe im Acetylaurin, in der Acetylrosolsäure und im Acetylbenzaurin in ihrer Reactionsfähigkeit stärker influenziert sein soll als in den Carbinolen des Bittermandelölgrüns oder Krystallvioletts.

Um nun den Fall möglichst einfach zu gestalten, haben wir uns der Untersuchung des Triphenylcarbinols zugewendet. Wir konnten bei diesen Studien die Überzeugung gewinnen, dass die Reactionen des Triphenylcarbinols keineswegs von einer einheitlichen Formel ausgehend erklärt werden können, so dass man auch hier mit tautomeren Formen rechnen muss.

1 Berl. Ber,, XXXIII, 3356. 
Das Triphenylcarbinol besitzt, wie zum Theile bereits bekannt und außerdem im experimentellen Theile gezeigt werden soll, eine Gruppe, welche in vielen Beziehungen so reagiert wie die Hydroxylgruppe in einzelnen Phenolen und namentlich in Säuren. Dieser Charakter erweist sich klar bei der überaus leichten Bildung der Alkyläther mit Alkohol und Schwefelsäure, mit Alkohol und dreiprocentiger Salzsäure und sogar mit Alkohol und verdünnter Essigsäure. Diesen Eigenschaften steht aber scharf die Indifferenz des Triphenylcarbinols gegen Alkalien gegenüber. Hemilian hat gefunden, dass Alkalien auf das Carbinol gar nicht einwirken, und diese Beobachtung können wir voll und ganz bestätigen. Aus einer alkoholischen Lösung von Ätzkali und Triphenylcarbinol lässt sich letztere Verbindung durch Wasser vollständig rein mit dem richtigen Schmelzpunkte ausfällen und auch mit Äther ausschütteln. Zwar gibt Hemilian an, dass er mit Natrium in einer Lösung des Carbinols in Toluol eine Natriumverbindung als. Ausscheidung erhalten hat; diese Verbindung ist jedoch nach seinen Angaben stark hygroskopisch und zerfällt mit Wasser sofort in Natriumhydroxyd und Carbinol. Außerdem konnten wir constatieren, dass die Lösung dieser Substanz in Toluol, mit Jodalkyl behandelt, keinen Alkyläther liefert, sondern reines Triphenylcarbinol. ${ }^{1}$

Mit diesen Thatsachen ist das merkwürdige Verhalten des Carbinols noch lange nicht erschöpft.

Wie die Acetylaurine lässt sich auch das Carbinol sehr leicht reducieren, und man erhält mit Zink in Eisessig Triphenylmethan.

Eine nahe Beziehung mit dem Fischer'schen, respective Rosenstiehl'schen Äther ergibt das Verhalten des Äthyläthers des Carbinols gegen Säuren, beziehungsweise Alkalien. Gegen letztere erweist sich der Äther vollkommen resistent, während schon ganz verdünnte Säuren denselben verseifen.

Sehr merkwürdig und interessant ist die leichte Überführbarkeit der Acetyl- und Äthyläther ineinander. Das Acetyl-

1 Über ähnliche Beobachtungen bei den Alkyloxantrhanolen siehe: Liebermann, Ann. Chem. Pharm., 212, 115. 
derivat, aus Alkohol umkrystallisiert, liefert quantitativ den Äthyläther, während letzterer, mit Essigsäureanhydrid oder Acetylchlorid behandelt, in die Acetylverbindung übergeht.

Schließlich möge noch die bemerkenswerte Thatsache erwähnt werden, dass die angebliche Hydroxylgruppe des Carbinols wohl mit Acetylchlorid, aber nicht mit Essigsäureanhydrid sich acetylieren lässt.

Übersieht man diese verschiedenen Beobachtungen, so wird man zugeben müssen, dass sie, wenn überhaupt, so doch nur außerordentlich schwierig von einer einheitlichen Formel aus erklärt werden können, zumal da die erwähnten Reactionen nahezu quantitativ verlaufen. Es wird sich daher infolge dessen vielleicht empfehlen, auch beim Triphenylcarbinol selbst zwei tautomere Formeln anzunehmen. Wollte man dies umgehen, so müsste man in diesem Körper einen Hydroxylrest sui generis annehmen, der in seinen Eigenschaften an verschiedene Körperclassen erinnert und sich mit keiner der bekannten Arten der Hydroxylgruppe ganz in Einklang bringen lässt.

Was nun die Art der Tautomerie betrifft, so würden wir mit Rücksicht auf die Analogie einer Formel den Vorzug geben, welche direct sich von der tautomeren Form des Acetylaurins ableiten lässt.

Wir hätten dann beim Triphenylcarbinol folgende Configurationen anzunehmen:

I.

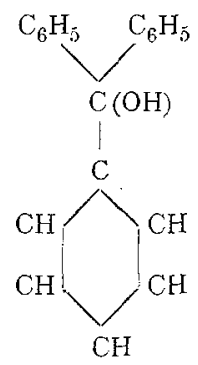

II.

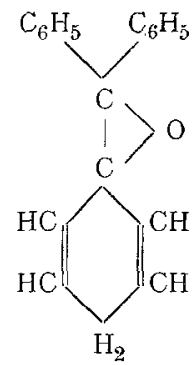

Wir können uns nicht verhehlen, dass sich die Beweise für die Form II schwer werden erbringen lassen, da wir über das Verhalten einer derartigen Verbindung nur Vermuthungen aufstellen können. Sicher ist aber, dass die Form II die leichte 
Reducierbarkeit, die Indifferenz gegen Alkali und die merkwürdige Isomerie bei den Acetylaurinen leicht erklären lässt. Das Hauptgewicht soll und muss aber vorerst auf die Thatsache des merkwürdigen Verhaltens und auf die Wahrscheinlichkeit einer Tautomerie gesetzt werden.

\section{Acetylrosolsäure.}

Rosolsäure, mit dem gleichen Gewichte an Natriumacetat und mit Essigsäureanhydrid im Überschusse gekocht, liefert ein durch Wasser ausfällbares Acetylproduct, welches im Vacuum getrocknet wurde. Aus Alkohol umkrystallisiert, ließ sich der Körper in zwei Fractionen trennen, eine in kaltem Alkohol fast unlösliche, bei 167 bis $168^{\circ}$ (uncorr.) schmelzende und eine leicht lösliche, zu deren Gewinnung man den Alkohol fast ganz abdunsten musste. Letztere zeigte den Schmelzpunkt 144 bis $146^{\circ}$ und entstand nur in äußerst geringen Quantitäten.

Die Analyse des schwer löslichen Productes wies, wie vorherzusehen war, deutlich auf ein Triacetat hin.

I. $0 \cdot 1870 \mathrm{~g}$ Substanz, bei $100^{\circ}$ getrocknet, gaben $0.4775 \mathrm{~g}$ Kohlensäure und $0.0830 \mathrm{~g}$ Wasser.

In 100 Theilen:

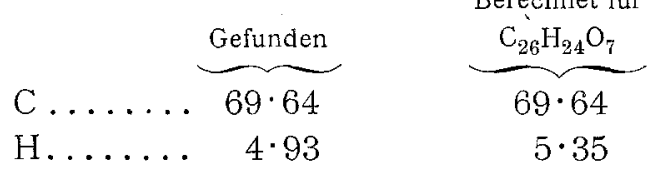

Die leicht lösliche Fraction entsteht leider nur in so geringer Menge, dass es nicht möglich war, dieselbe als ein zweites Triacetat genau zu charakterisieren. Doch konnte die Verbindung immer von constantem Schmelzpunkt erhalten werden, und die Analogie mit dem Acetylaurin spricht wohl sehr für die Natur derselben als ein isomeres Acetylderivat.

\section{Benzaurindiacetat.}

Döbner beschreibt ${ }^{1}$ ein Acetylproduct des von ihm dargestellten Benzaurins oder Phenolbenzeins, welches er nach 
sorgfältiger Reinigung des Farbstoffes durch Auflösen in Natriumbisulfit und Wiederausfällen mit Säure erhalten hat, indem er die rothen, metallglänzenden Krusten mit Essigsäureanhydrid auf $100^{\circ}$ im Einschmelzrohre erhitzte und das gebildete Product durch Wasser ausfällte.

Wenn man aber der Beschreibung des Autors folgt, so gelangt man $z u$ einem Körper, der bei 144 bis $146^{\circ}$ schmilzt und diesen Schmelzpunkt beim Umkrystallisieren aus Alkohol constant beibehält. Der von Döbner beschriebene Körper schmilzt dagegen bei 116 bis $119^{\circ}$, und es gelang nur in einem von zehn Fällen, ihn in sehr schlechter Ausbeute darzustellen. Dagegen gelangten wir immer zu dem anderen oben erwähnten Acetylderivat, gleichgiltig $o b$ die Acetylierung im Rohre, bei niederer oder hoher Temperatur, mit oder ohne Natriumacetat, mit gereinigter oder roher Substanz vorgenommen wurde. Der erhaltene Körper wurde, wie bemerkt, aus Alkohol umkrystallisiert, im Vacuum getrocknet und lieferte folgende Zahlen:

I. $0.1909 \mathrm{~g}$ Substan $z$ gaben $0.5139 \mathrm{~g}$ Kohlensäure und $0.0935 \mathrm{~g}$ Wasser.

II. $0.1911 \mathrm{~g}$ Substanz gaben $0.5160 \mathrm{~g}$ Kohlensäure und $0.0955 \mathrm{~g}$ Wasser.

III. $0.1855 \mathrm{~g}$ Substanz gaben $0.4974 \mathrm{~g}$ Kohlensäure und $0.0859 \mathrm{~g}$ Wasser.

IV. $0 \cdot 2032 g$ Substanz neutralisierten bei der Wenzel'schen Acetylbestimmung

$11 \cdot 4 \mathrm{~cm}^{3} 1 / 10^{-n o r m a l e}$ Kalilauge.

V. $0 \cdot 2142 g$ Substanz neutralisierten bei der Wenzel'schen Acetylbestimmung $11: 7 \mathrm{~cm}^{3} 1 / 10^{-n o r m a l e}$ Kalilauge.

In 100 Theilen:

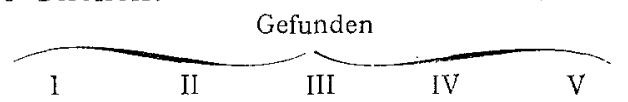

Berechnet für

$3 \cdot 60$

$73 \cdot 13$

- -

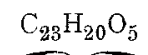

C ......73:40

$5 \cdot 55$

$5 \cdot 14$

- -

$73 \cdot 40$

$\mathrm{H} \ldots \ldots .5 \cdot 44$

$\mathrm{CH}_{3} \mathrm{CO} \ldots$ - $\quad$ - $\quad$ - $24 \cdot 1 \quad 23 \cdot 5 \quad 22 \cdot 9$

$\mathrm{CH}_{3} \mathrm{CO} \ldots$ - $\quad$ - $\quad$ - $24 \cdot 1 \quad 23 \cdot 5 \quad 22 \cdot 9$

$5 \cdot 34$

Diese Analysen weisen also nothwendig auf ein Diacetat, ebenso wie es Döbner für seinen Körper angibt. In der That wurde der von uns dargestellte Körper noch schärfer als durch die Elementaranalyse durch sein Verhalten bei der Reduction charakterisiert.

Wenn man nämlich das genannte Acetylproduct in essigsaurer Lösung mit Zinkstaub kocht, hierauf das Zink abfiltriert und mit heißem Eisessig nachwäscht, so kann man aus der 
Essigsäure mittels Wassers einen Körper ausfällen, dessen Schmelzpunkt nach oftmaligem Umkrystallisieren aus Alkohol bei 108 bis $110^{\circ}$ constant bleibt.

Dieser Eigenschaft und der Elementaranalyse zufolge erweist sich die Substanz als identisch mit dem von Rus sanow ${ }^{1}$ dargestellten Diacetat des Dioxytriphenylmethans, welches der genannte Autor aus Benzaldehyd und Phenol erhalten hat.

I. $0.1920 \mathrm{~g}$ vacuumtrockener Substanz gaben $0.5394 \mathrm{~g}$ Kohlensâure und $0.0970 \mathrm{~g}$ Wasser.

II. $0.1835 g$ vacuumtrockener Substanz gaben $0.5158 \mathrm{~g}$ Kohlensäure und $0.0966 \mathrm{~g}$ Wasser.

In 100 Theilen:

\begin{tabular}{|c|c|c|}
\hline \multicolumn{2}{|c|}{ Gefunden } & Berechnet für \\
\hline I & If & $\mathrm{C}_{23} \mathrm{H}_{20} \mathrm{O}_{4}$ \\
\hline $.76 \cdot 62$ & $76 \cdot 66$ & $76 \cdot 62$ \\
\hline$\ldots 5 \cdot 60$ & $5 \cdot 92$ & $5 \cdot 60$ \\
\hline
\end{tabular}

Von dem einmal erhaltenen Acetylproducte vom Schmelzpunkte 116 bis $119^{\circ}$ können wir nur sagen, dass dessen Schmelzpunkt constant blieb. Eine weitere Untersuchung war wegen der geringen Menge nicht möglich.

\section{Methyläther des Bittermandelölgrüns.}

Schon in einer früheren Publication von $\mathrm{O} . \mathrm{Fischer}{ }^{2}$ ist ein Äthyläther des Bittermandelölgrüns erwähnt, auf welchen der Autor bei Gelegenheit der Herstellung des entsprechenden Methyläthers ${ }^{3}$ zurückkommt. Nicht unwichtig erscheint bei der Herstellung dieses Äthers die Umkrystallisation aus einem Methylalkohol, dem etwa $0 \cdot 1 \%$ Ätzkali zugesetzt wird. Nur auf diese Weise gelang es uns, den Körper auf den von O. Fischer angegebenen Schmelzpunkt zu bringen und ihn einer Methoxylbestimmung zu unterwerfen.

$0.2450 \mathrm{~g}$ über Kali getrockneter Substan $z$ gaben nach $\mathrm{Z}$ eise $10 \cdot 1560 \mathrm{~g}$ Jodsilber.
1 Berl. Ber., XXII, S. 1944.
2 Lieb. Ann., 206, 132.
3 Berl, Ber., XXXIII, 3356 . 
In 100 Theilen:

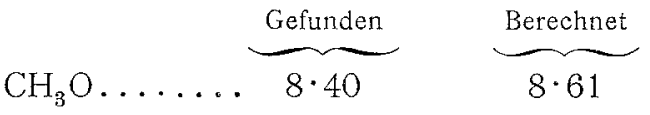

Es scheint somit sichergestellt, dass wir es hier mit einem ätherartigen Triphenylcarbinolderivate $z u$ thun haben, wie es beim Aurin herzustellen nicht möglich war.

\section{Äthyläther des Krystallvioletts.}

Dieselbe Erfahrung machten wir beim Äthyläther des Hexamethyltriamidotriphenylcarbinols. Der Körper, der genau

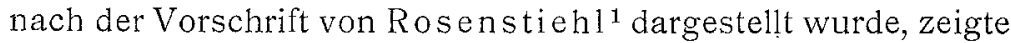
nach mehrmaligem Umkrystallisieren aus heißem, etwas kalihaltigem Alkohol den constanten Schmelzpunkt 143 bis $145^{\circ}$. Die Äthoxylbestimmung sprach deutlich für einen Äthyläther. $0.1615 \mathrm{~g}$ über Kali getrockneter Substanz lieferten nach Z eis el $0.0817 \mathrm{~g}$ Jodsilber.

In 100 Theilen:

$$
\mathrm{C}_{2} \mathrm{H}_{5} \mathrm{O} \ldots \underbrace{\text { Gefunden }}_{9 \cdot 68} \quad \underbrace{\text { Berechnet }}_{10 \cdot 79}
$$

Da wir in der Literatur keine Analysen von Rosenstiehl und auch keine Angaben über den Schmelzpunkt vorfanden, lag-die Möglichkeit einer Identificierung unserer Substanz mit seiner Verbindung nicht vor.

Wir gehen nun zu unseren Versuchen über das

\section{Triphenylcarbinol}

über. Wir erhielten dasselbe in guter Ausbeute nach dem Oxydationsverfahren E. und $\mathrm{O}$. Fischers ${ }^{2}$ und krystallisierten es aus etwa 80 procentigem Alkohol um. So konnten wir schon nach einmaliger Reinigung ein analysenreines Product erhalten, und zwar aus $15 \mathrm{~g}$ Triphenylmethan etwa 10 bis $12 \mathrm{~g}$ Carbinol. $0.1971 \mathrm{~g}$ Substanz, bei $100^{\circ}$ getrocknet, gaben $0.6343 \mathrm{~g}$ Kohlensäure und $0.1150 \mathrm{~g}$ Wasser.

1 Comptes rendus, 120 , S. 192 ,

2 Berl. Ber., XIV, 1944. 
In 100 Theilen:

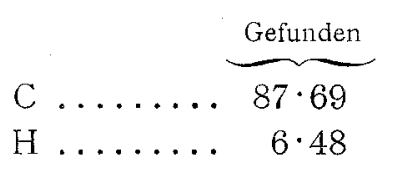

Berechnet für

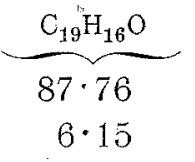

Hiebei wäre noch zu bemerken, dass das Carbinol, so leicht auch verschiedene Gruppen einführbar sein mögen, umgekehrt ganz indifferent ist, nicht nur gegen Kali, wie schon früher bemerkt wurde, sondern auch gegen Ammoniak. Die Amidogruppe trat weder beim Kochen mit starkem Ammoniak, noch mit festem Chlorzinkammoniak ein.

\section{Äthyläther des Triphenylcarbinols.}

Hemilian ${ }^{x}$ beschreibt die Darstellung des Äthyläthers des Triphenylcarbinols aus Alkohol und dem Chloride, welches seinerseits aus Carbinol und Phosphorpentachlorid entsteht. Inzwischen ist der Äther auch durch directe Esterification mit zehnprocentiger Schwefelsäure dargestellt worden. ${ }^{2}$ Diesem Verfahren schlossen sich unsere Versuche an, die ergaben, dass der Äthyläther auch mit dreiprocentiger alkoholischer Salzsäure entsteht, und zwar in sehr guter Ausbeute. Vorgreifend müssen wir hier bemerken, dass auch durch Behandeln des Acetylderivates mit Alkohol ganz leicht der reine Äthyläther erhalten werden kann.

Durch eine Äthoxylbestimmung konnte nachgewiesen werden, dass wir es auch hier mit einem echten Äther des Carbinols zu thun hatten.

$0.2702 g$ vacuumtrockener Substanz gaben nach Z eis e $10.1995 g$ Jodsilber.

In 100 Theilen:

$$
\mathrm{C}_{2} \mathrm{H}_{5} \mathrm{O} \ldots \ldots \frac{\text { Gefunden }}{14 \cdot 2} \quad \frac{\text { Berechnet }}{15 \cdot 6}
$$

Was die Herstellungsweise des Äthers betrifft, so ist die ebenerwähnte mit Alkohol und Salzsäure allen bisher

\footnotetext{
1 Berl. Ber., VII, 1204 u. ff.

2 Cbl., 1897, II, S. 408.
} 
gebrauchten vorzuziehen. Die Ausbeute ist fast quantitativ. Aus dem Alkohol krystallisiert der im kalten Lösungsmittel schwer lösliche Äther in großen glasglänzenden Krystallen aus, die Herr Hofrath Prof. V. v. Lang zu messen die Güte hatte.

»Die gut ausgebildeten, farblosen Krystalle gehören in das monokline System und zeigen die Flächen 001, 010, 110, 011.

Ein Theil derselben hat eine dem Gips ähnliche Ausbildung, andere aber sind tafelförmig durch das Vorherrschen der Fläche 001.

Elemente $a: b: c=0.6257: 1: 0 \cdot 5056, a c=120^{\circ} 41^{I}$.

Es wurde nun das Verhaiten dieses Äthers gegen alle diejenigen Agentien geprüft, von denen man erwarten durfte, dass sie auf die Alkoxylgruppe einwirken würden. Gegen siedendes Wasser verhielt sich der Körper beständig; da er bei 78 bis $80^{\circ}$ schmilzt, so konnte man diese seine Beständigkeit gegen kochendes Wasser daran wahrnehmen, dass er geschmolzen blieb. Ebenso beständig war er gegen alkoholische Kalilauge; nach dem Ausäthern zeigte der aus dem Äther auskrystallisierende Rückstand wieder den Schmelzpunkt 78 bis $80^{\circ}$ und den charakteristischen Habitus der Triphenylcarbinäthylätherkrystalle.

Anders jedoch wirken verdünnte Säuren auf ihn ein. Die Substanz, mit wässeriger sechsprocentiger Salzsäure gekocht, schmolz beim Erhitzen der Flüssigkeit, nach einer Viertelstunde aber erstarte sie während des Kochens zu einem krystallinischen Kuchen, der den Schmelzpunkt des Triphenylcarbinols. $\left(159^{\circ}\right)$ aufwies. Ein anderer Versuch zeigte, dass der Äther nicht nur mit Acetylchlorid, ${ }^{1}$ sondern auch mit Essigsäureanhydrid in das Acetylproduct des Carbinols übergeht. Beim Kochen des Äthyläthers mit Anhydrid und nachherigen Abdestillieren der Flüssigkeit im Vacuum blieb ein öliger Rückstand zurück, der eine negative Methoxylbestimmung lieferte und mit Wasser gekocht, in Triphenylcarbinol übergieng.

Ein Versuch, bei dem Natriumacetat der Lösung noch hinzugefügt wurde, verlief ganz analog.

1 Lieb. Ann. 227, 116. 


\section{Essigsäureester des Triphenylcarbinols.}

Allen und Kölliker ${ }^{1}$ gelang die Darstellung des Essigsäureesters des Triphenylcarbinols (Schmelzpunkt 98 bis $99^{\circ}$ ) aus dem Hemilian'schen Methyläther mit Acetylchlorid. Sowohl aus dem. Entweichen von Alkylchlorid, als aus der Analyse ergab sich die Constitution des genannten Körpers.

Hemilian selbst hatte versucht, das Triphenylcarbinol der directen Acetylierung durch Destillation mit Anhydrid zu unterwerfen, was ihm aber nicht gelang. Dagegen erhielt er mit Acetylchlorid eine strahlig-krystallinische Masse, die sich unter Essigsäureentbindung an der Luft zersetzte. Bei der Wiederholung dieses Versuches gelangten wir zur Erkenntnis, dass die Zersetzlichkeit des Körpers nicht so groß sei, als $\mathrm{He}$ milian angibt. Unter Wasser geht er allerdings schon innerhalb weniger Stunden in Carbinol über, an der Luft aber bewahrt er seinen Schmelzpunkt selbst durch etwa zehn Tage constant. Daher empfahl es sich, die Darstellung des Körpers, ohne den Umweg über den Alkyläther, direct aus Triphenylcarbinol und Acetylchlorid vorzunehmen.

Bei den Versuchen, denselben zu reinigen, stieß man auf Schwierigkeiten. Allen und Kölliker geben als Krystallisationsmittel siedenden Äther an, doch ist er schon in kaltem Äther so leicht löslich, dass große Verluste unvermeidlich wären.

Am besten bewährte sich die Auflösung in wenig heißem Benzol und nachherige Ausfällung mit Petroläther. Als wir den Körper aus Alkohol umkrystallisieren wollten, stießen wir auf die merkwürdige Erscheinung, dass sich derselbe quantitativ in Äthyläther umsetzt, wie schon weiter oben bemerkt wurde.

Wir können also zusammenfassend den Austausch von Äthyl gegen Acetyl mittels Acetylchlorid, Essigsäureanhydrid und Natriumacetat oder Anhydrid allein, den Austausch von Acetyl gegen Äthyl schon beim Kochen mit Alkohol constatieren. Die leichte Austauschbarkeit der Acetyl- und Äthylgruppe ist also charakteristisch für das Carbinol und der jeweilige Eintritt

\footnotetext{
1 Ann. Chem. Pharm., 227, 116.
} 
einer solchen Gruppe beruht nur auf einer Massenwirkung des betreffenden Agens.

Es war von Interesse, festzustellen, ob die Acetylierung des Carbinols auch mit Essigsäureanhydrid vor sich geht. Ein directer Versuch ergab nach dem Abdestillieren des Anhydrids. einen Körper, der sich durch den Schmelzpunkt als Carbinol erwies.

Der Versuch mit Essigsäureanhydrid und Natriumacetat ließ sich nur auf Umwegen bewerkstelligen, weil die Trennung des Natriumacetats vom eigentlichen Reactionsproducte nur mittels Wassers möglich wäre, wobei sich das eventuell entstandene Acetylderivat verseifen würde. Mit Rücksicht darauf haben wir das Reactionsgemisch nach Abdestillieren des Essigsäureanhydrids direct mit Alkohol behandelt. Vorausgesetzt, dass sich das Acetylderivat gebildet hätte, würde sich dasselbe durch Kochen mit Alkohol in das Äthylderivat umwandeln, und letzteres ließe sich dann mit Wasser leicht vom Natriumacetat trennen.

In der That erhielten wir auf diesem Wege einen Körper, dessen Schmelzpunkt deutlich auf ein Gemisch von Äthyläther und Carbinol hinwies. Die Äthoxylbestimmung lieferte Daten, die auf einen Gehalt von $20 \%$ an Äthyläther hingedeutet haben.

Diesem Versuche wurde aber sehr viel an Beweiskraft entzogen, als wir uns überzeugt hatten, dass das Carbinol mit Alkohol und verdünnter Essigsäure sich sehr leicht alkylieren lässt. Man müsste diese Beobachtung infolgedessen mit größeren Cautelen wiederholen, um jede Spur von Essigsäure zu vermeiden, und dazu hat es uns an Material gemangelt. Immerhin erscheint uns selbst die wenn auch nur theilweise Acetylierung: mit Essigsäureanhydrid und Natriumacetat sehr zweifelhaft.

\section{Reduction des Triphenylcarbinols.}

Eine Relation zwischen dem Carbinol einer- und substituierten Triphenylmethanen anderseits bildet die Reducierbarkeit zu Triphenylmethan.

Hemilian constatierte, dass sich das Triphenylcarbinol gegen Natriumamalgam unverändert zeige. Dagegen gelang uns die Reduction mit Zink und Eisessig bei mehrstündigem Kochen. 
614 J. Herzig und P. Wengraf, Carbinolverbindungen etc.

$0 \cdot 1903 g$ vacuumtrockener Substanz gaben $0 \cdot 6490 \mathrm{~g}$ Kohlensäure und $0 \cdot 1060 \mathrm{~g}$ Wasser.

In 100 Theilen:

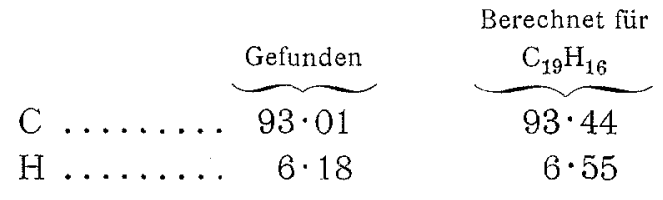

Die Analyse zeigte also, dass in der That Triphenylmethan vorliegt, mit welcher Thatsache der beobachtete Schmelzpunkt (92 bis $\left.93^{\circ}\right)$ vollkommen übereinstimmt.

Schließlich sei es uns an dieser Stelle gestattet, Herrn Hofrath Prof. Dr. V. v. Lang für seine Krystallmessung unseren besten Dank auszusprechen. Den Höchster Farbwerken sind wir für die Überlassung einiger Präparate sehr zu Dank verpflichtet. 